\title{
Additional intranasal oxytocin to escitalopram improves depressive symptoms in resistant depression: An open trial
}

\author{
G. Scantamburlo a, , M. Hansenne b, V. Geenen c, J.J. Legros d, M. Ansseau a
}

a Department of Psychiatry, Psychoneuroendocrinology Unit, University of Liège, Liège, Belgium

${ }^{b}$ Department of Cognitive Sciences, Faculty of Psychology, University of Liège, Liège, Belgium

c Immunoendocrinology Center, GIGA3, University of Liège, Liège, Belgium

${ }^{d}$ Departement of Endocrinology, Psychoneuroendocrinology Unit, University of Liège, Liège, Belgium

KEYWORDS: Major depression Intranasal oxytocin Anxiety

\begin{abstract}
The aim of this open trial was to assess the antidepressant/anxiolytic effects of oxytocin used as an adjunct to antidepressant in treatment-resistant depression. Fourteen patients, who have not responded to $40 \mathrm{mg}$ of escitalopram, received intranasal synthetic oxytocin during 4 weeks, in association with antidepressant. This is the first open trial study suggesting OT in association with escitalopram significantly reduced scores on Hamilton Depression Rating Scale.
\end{abstract}




\section{Introduction}

The nonapeptide oxytocin (OT) is implicated in social affiliation, sexual and maternal-infant bonding, anxiety, mood, feeding control and memory. It is also involved in the regulation of emotions, and improves cooperation altruism, and trust in a variety of experimental context [1].

There are also speculations that some of the symptoms commonly reported in depression (social withdrawal, reduced appetite, cognitive impairment) may reflect dysregulation of OT functions. There are relatively few data on plasma OT levels in depression. Discrepancies have been reported. Periphery OT levels were found to be reduced, increased or unchanged [19,20]. Cyranowski et al. (2008) found a dysregulated pattern of peripheral oxytocin among depressed woman $(n=17)$ compared to controls $(n=17)$. Previously, we reported an association between plasma OT levels and anxiety in patients with major depression [22]. A polymorphism of oxytocin receptor (OTR) has been associated with major depression (Thompson et al., 2011). Recently, no association was observed between OTR genes (rs 53576, rs 2254298) and treatment resistance, response and remission in a sample of 268 patients [14].

Preclinical evidence indicates that OT can influence activity of serotonin system, which may related to the antidepressant effects of SSRIs [9]. Emiliano et al. [5] have demonstrated overlap of OT-labelled neurons and 5-HTT-labelled fibers in the parvicellular, magnocellular, dorsal and posterior subdivisions of the PVN and provided neuroanatomic support for the idea that SSRIs therapeutic effects on social affiliation and anxiety may be mediated in part through components of the OT system.

Many data suggest a role for OT as an endogenous antidepressant/anxiolytic hormone [26] and support the idea that stimulation of OT receptors inhibits the hypothalamo-pituitary- adrenal (HPA) axis [17]. The oxytocin and dopaminergic systems may act in concert to reduce anxiety in response to social and environmental stressors. Heinrichs et al. [8] have demonstrated that OT insufflation (24 I.U.) and social support during public speaking reduce stress responses. OT further has stress-protective effects [28]. Interestingly, OT decreases amygdala reactivity to aversive, threat-related faces [2] in males, but increases amygdala reactivity to similar faces in females [10].

Implicit in these results is potential therapeutic opportunity of targeting the central oxytoninergic system in the development of novel anxiolytics for humans. The pathophysiology of stress- related diseases, such as depression whereas anxiety disorders, includes both endogenous/genetic predisposing factors and a dysregulated response to stress, and normalizing the HPA axis is one of the major targets of recent treatments [9]. The aim of the present open trial study was to assess the effect of intranasal OT - in association to escitalopram - in patients with have not responded to escitalopram, as suggested in a previous case report [23]. 


\section{Methods}

The study was performed in 14 outpatients with major depression, representing consecutive admissions to the Biological Psychiatry and Psychoneuroendocrinolgy Unit of the University of Liège, Belgium. The diagnosis of major depressive disorder was based on the DSM-IV criteria and the self-questionnaire from the Mini International Neuropsychiatric Interview. Moreover, patients had a score of at least 17 on the 17-item Hamilton Depression Rating Scale (HDRS-17). Patients have required to have experienced treatment-resistant depression as defined by failure of adequate trials of two differents classes of major antidepressants at the recommanded maximun doses. Patients had at least a 2-year history of resistant depression. All patients, who have not responded to $40 \mathrm{mg}$ of escitalopram at least during 8 weeks, received intranasal synthetic oxytocin (Syntocinon1, 1 puff per nostril, each with 4 I.U., twice daily) (Novartis, Switzerland) during 4 weeks, as a add-on treatment to $40 \mathrm{mg}$ of escitalopram. The daily dosage of 16 I.U. has been chosen according to usual range in other psychiatric diseases. Moreover, a recent study has documented that salivary OT levels in normal subjects remained similarly elevated for up to $7 \mathrm{~h}$ of 16 or 24 I.U [27]. Among the 6 women, 4 were premenopausal and 2 were postmenopausal. All premenopaus women were investigated during the first phase of the menstrual cycle as assessed by the patient-reported date of last menstruation. Patients with irregular menses were excluded. All patients were free of medical illness as evidence by history, medical examination, ECG, chest X-ray, EEG, and routine laboratory tests. Except their current antidepresant, they had also been free of other drugs, including benzodiazepines, for at least 2 weeks at the time of the study. We used and provided standardised instructions and regular monitoring to train participants in administration. Individuals with common cold that result in significant nasal congestion were not included in the study. The protocol was approved by the Ethical Committee of the University of Liège, Belgium. All patients were fully informed of the purpose of the study and gave written consent.

Clinical assessment was perform before the first day of intranasal oxytocin treatment, and repeated each week for four weeks. Depressive and anxiety severity was assessed by the Hamilton Depressive Rating Scale (HDRS-17, Hamilton, 1967), and by the State-Trait Anxiety Inventory (STAI-A State; [24]), respectively. Clinical Global Impressions Severity of Illness scale (CGI-S, [6]) was used to assess the global illness severity. Quality of life was assessed by the Quality of Life Enjoyment and Satisfaction Questionnaire (Q-LES-Q, [4]).

All the statistical analyses were performed with Statistica (10.1) for Windows. Repeatedmeasures analysis of variance (ANOVA) on HDRS-17, STAI-A, CGI-S, and Q-LES-Q was conducted with time as within-subject factor. Tukey post-hoc comparisons were used to compare the clinical data for the different times. No side effects were reported following treatment with chronic OT.

\section{Results}

The sample included 6 women with a mean age of 48.5 years (S.D. $=13.9)$ and 8 men with a mean age of 44.75 years (S.D. $=9.1$ ). Table 1 details patient baseline clinical status. No statistical differences were observed between wash out period and baseline. 
Table 1. Patients clinical status at baseline.

Measure $(\mathrm{n}=14)$

Severity rating scale scores, mean (SD)

\begin{tabular}{llr}
\hline HDRS-17 & $\rightarrow$ & $26,13(5,99)$ \\
STAI-A & $\rightarrow$ & $64,88(15,88)$ \\
CGI-S & $\rightarrow$ & $4,29(0,95)$ \\
Q-LES-Q & $\rightarrow$ & $1,75(0,76)$
\end{tabular}

SD: standard deviation

Post-hoc analysis showed significant differences between day 1 and day 8 (HDRS-17, $\mathrm{P}<$ 0.001; STAI-A, P = 0.04; CGI-S, P $=0.02$ and Q-LES-Q, $P=0.046$ ), between day 1 and day 15 (HDRS-17, $\mathrm{P}<0.01$; STAI-A, $\mathrm{P}=0.015$; CGI-S, $\mathrm{P}=0.03$ and Q-LES-Q, $\mathrm{P}=0.044$ ), between day 1 and day 21 (HDRS-17, $\mathrm{P}=0.016$; CGI- $\mathrm{S}<0.01$ ), and between day 1 and day 28 (HDRS, $\mathrm{P}<$ 0.01; STAI-A, $\mathrm{P}=0.04$; CGI-S, $\mathrm{P}<0.01$ and Q-LES-Q, $\mathrm{P}=0.014$ ) (Fig. 1). The CGI-S ratings decreased from initial visit mean of 4.3 (S.D. $=0.95)$ to a mean of $2($ S.D. $=1.5)$ (Fig. 2).

\section{Discussion}

OT has been proposed as a potential treatment for a wide range of brain disorders: addiction, anxiety disorders, autism-spectrum disorders, borderline personality disorders, mood disorders and schizophrenia [11]. To our knowledge, this is the first open trial study suggesting that OT in association with escitalopram significantly reduced scores on HDRS-17 and STAI-A in addition to CGI-improvement scale. Moreover, patients showed improved positive evaluations of quality of life. OT was well-tolerated and no adverse effects were reported. The main limitation of this open trial is the small sample of subjects with mixed gender and the lack of a control group. As OT was given in parallel with escitalopram, the observed effects could be due to this combination or to the placebo effect.

There are limited studies exploring the effect of intranasal OT administration in major depression. Pincus et al. [21] have shown that depression is associated with increased paralimbic activity during emotional mental attribution of others, appearing to be distincly modulated by OT when compared to healthy controls. Recently, in mothers with a diagnosis of postnatal depression, OT administrations have been link to improvement of the perception of the relationship with the baby [12]. In addition, intranasal OT has impeded the ability to ignore task-irrelevant facial expressions of sadness in students with depressive symptoms [3].

OT plays a leading role in social neuroscience. The possible molecular mechanisms underlying antidepressant/anxiolytic effect of OT could be due to the induction of hippocampal neurogenesis, the modulation of amydgala activity and the inhibitory action on the HPA axis [13]. OT has a potent antidepressant effect following its secretion in the CNS, including the hippocampus and amygdala. In mice, OT induces phosphor- ylation of CREB throught activation of MAP kinase signaling and induces neuronal plasticity in the hippocampus [13]. BDNF gene expression was also found to be up-regulated in primary culture neurons treated with OT by microarray analysis [18]. Furman et al. [7] have shown variant in OTR associated with amygdala 
volume. The effect of the OXTR polymorphism on amygdala volume may be mediated, in part, by exposure to corticosteroids, which with chronic exposure may lead to atrophy of amygdala dendrites. It is possible that the efficacy of SSRIs in restoring interest in social interactions is due, in part, to their actions on the reward circuit via the OT system [5]. The effects of antidepressants on OT levels has become a research subject. A study by Ozsoy et al. [19] suggested that serum OT levels were non affected by antidepressant use in patients with unipolar and bipolar depression. In contrast, there are other studies proposing that an increase in central OT levels may be one of the underlying causes for the activities of serotoninergic antidepressants [25].

Moreover, the behavioral relevance of intranasal OT in patients and its association with central nervous system neuropeptide availability and function remain unclear [15] and complex. Caution should remain in force. OT seems to promote aggression or other antisocial behavior in some circumstances. Its effects also appear to vary depending on a person's genetic makeup and psychosocial status [16]. In addition, Macdonald and Feifel [11] suggest that incertain clinical populations and contexts, OT has heterogeneous subjective effects which may include acute anxiogenesis.

Findings should be replicated and extended in a larger samples and a randomized placebocontrolled trials, controlling age, gender, and stage of menstrual cycle. We have not found an effect of sex in our study but the number of patients included was probably not sufficient to analyse for a sex-by-drug effect. Further neuroendocrine studies are needed to investigate the effects of OT or OT receptors selective agonists in additional clinical models to promote the development of psychopharmacology targeting central OT receptors.

Figure 1. HDRS-17 scores at days 1, 8, 15, 21 and 28 in depressive patients with intranasal synthetic OT, in association with antidepressant treatment

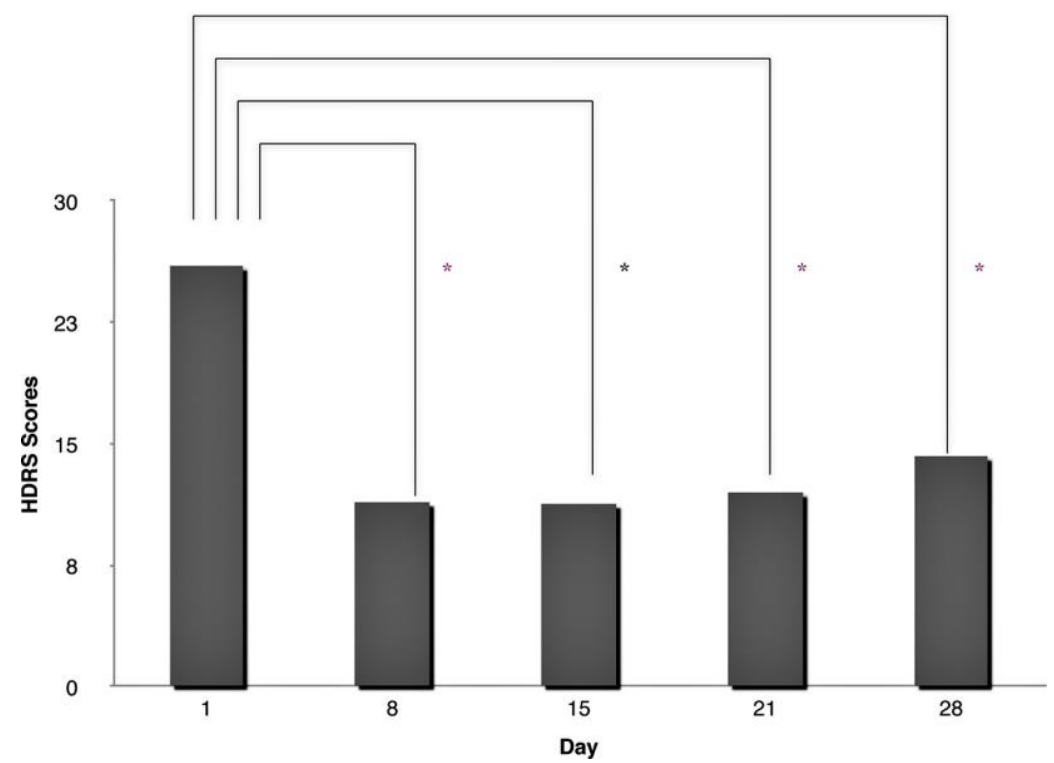

Figure 2. CGI-S scores at days 1, 8, 15, 21 and 28 in depressive patients with intranasal synthetic OT, in association with antidepressant treatment. 


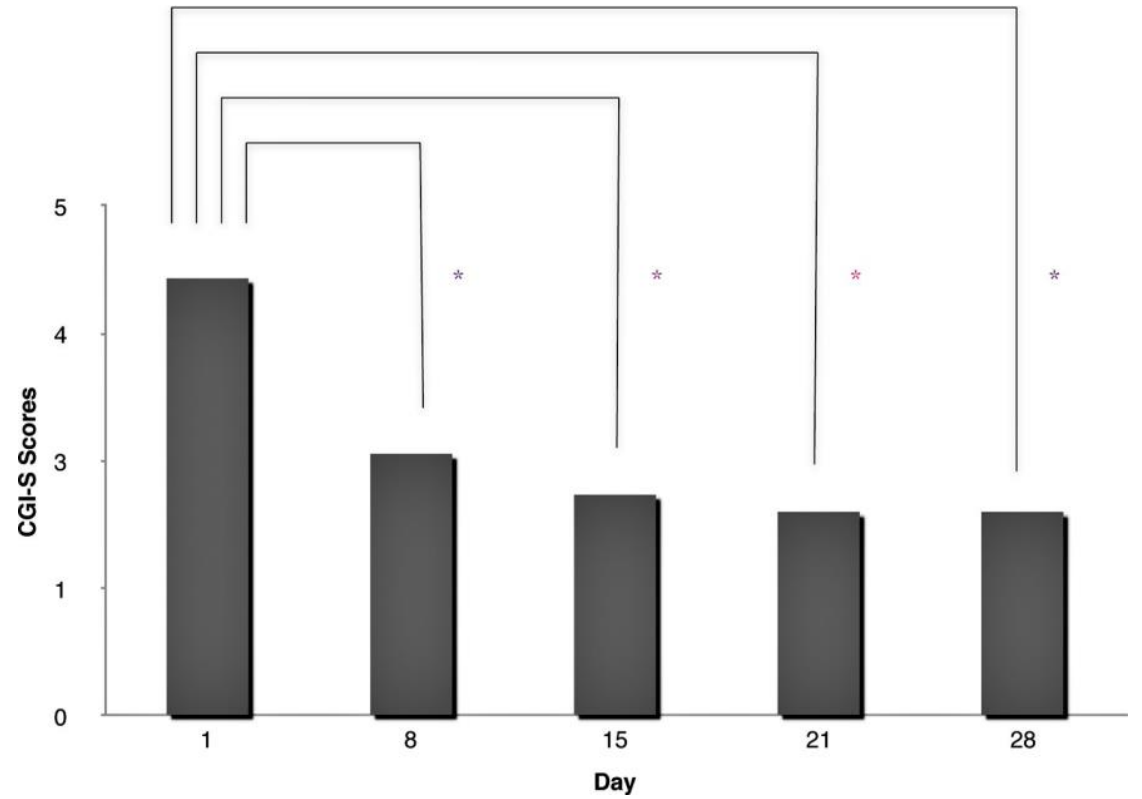

\section{Disclosure of interest}

The authors declare that they have no conflicts of interest concerning this article.

\section{Acknowledgements}

The authors gratefully acknowledge $\mathrm{C}$. Renard for her technical assistance and the patients.

Funding: Partial funding for the study was provided by Grant from the National Found of Scientifc Research (FRS-FNRS). 


\section{References}

[1] Cardoso C, Ellenbogen M, Linnen A. Acute intranasal oxytocin improves positive self-perceptions of personality. Psychopharmacology 2012;220: 741-9.

[2] Domes G, Lischke A, Berger C, Grossmann A, Hauenstein K, Heinrichs M, et al. Effects of intranasal oxytocin on emotional face processing in women. Psychoneuroendocrinology 2010;35:83-93.

[3] Ellenbogen MA, Linnen AM, Cardoso C, Joober R. Intranasal oxytocin impedes the ability to ignore taskirrelevant facial expressions of sadness in students with depressive symptoms. Psychoneuroendocrinology 2013;38:387-98.

[4] Endicott J, Nee J, Harrison W, Blumenthal R. Quality of life enjoyment and satisfaction questionnaire: a new measure. Psychopharmacol Bull 1993;29: 321-6.

[5] Emiliano A, Cruz T, Pannoni V, et al. The interface of oxytocin-labeled cells and serotonin. Neuropsychopharmacology 2006;11:1-12.

[6] Forkmann T, Scherer A, Boecker M, et al. The clinical global Impression scale and the influence of patient or staff perspective on outcome. BMC Psychiatry 2011;11(1):83.

[7] Furman DJ, Chen MC, Gotlib IH. Variant in oxytocin receptor gene is associated with amygdala volume. Psychoneuroendocrinology 2011;36:891-7.

[8] Heinrichs M, Baumgartner T, Kirschbaum C, Ehlert U. Social support and oxytocin interact to suppress cortisol and subjective responses to psychosocial stress. Biol Psychiatry 2003;54:1389-98.

[9] Keating C, Dawood T, Barton D, Lambert G, Tilbrook A. Effects of selective serotonin reuptake inhibitor treatment on plasma oxytocin and cortisol in major depressive disorder. BMC Psychiatry 2013;29(13):124.

[10] Lischke A, Gamer M, Berger C, Grossmann A, Hauenstein K, Heinrichs M, et al. Oxytocin increases amygdala reactivity to threatening scenes in females. Psychoneuroendocrinology 2012;37:1431-8.

[11] Macdonald K, Feifel D. Helping oxytocin deliver: considerations in the development of oxytocin-based therapeutics for brain disorders. Front Neurosci 2013;7:1-21.

[12] Mah BL, Van Ijzendoorn MH, Smith R, Bakermans-Kranenburg MJ. Oxytocin in postnatally depressed mothers: its influence on mood and expressed emotion. Prog in Neuropsychopharmacol Biol Psychiatr 2013;40:267-72.

[13] Matsuki M, Matsushita H, Tomizawa K, Matsui H. Oxytocin: a therapeutic target for mental disorder. J Physiol Sci 2012;62:441-4.

[14] Mendlewicz J, Crisafulli C, Calati R, Kocabas NA, Massat I, Linotte S, et al. Influence of COX-2 and OXTR polymorphisms on treatment outcome in treatment resistant depression. Neurosci Lett 2012;10(516):858.

[15] Meyer-Lindenberg A, Domes G, Kirsch P, Heinrichs M. Oxytocin and vasopressin in the human brain: social neuropeptides for translational medicine. Nat Rev Neurosci 2011;19(12):524-38.

[16] Miller G. The promise and perils of oxytocin. Science 2013;339:267-9.

[17] Legros JJ. Inhibitory effect of oxytocin on corticotrope function in humans: are vasopressin and oxytocin ying-yang neurohormones? Psychoneuroendocrinology 2011;26:649-55. 
[18] Okimoto N, Bosch OJ, Slattery DA, Pflaum K, Matsushita H, Wei FY, et al. RGS2 mediates the anxiolytic effect of oxytocin. Brain Res 2012;9(1453):26-33.

[19] Ozsoy S, Esel E, Kula M. Serum oxytocin levels in patients with depression and the effects of gender and antidepressant treatment. Psychiatry Res 2009;30(169):249-52.

[20] Parker KJ, Kenna HA, Zeitzer JM, Keller J, Blasey CM, Amico JA, et al. Preliminary evidence that plasma oxytocin levels are elevated in major depression. Psychiatry Res 2010;178:359-62.

[21] Pincus D, Kose S, Arana A, Johnson K, Morgan P, Borckardt J, et al. Inverse effects of oxytocin on attributing mental activity to others in depressed and healthy subjects: a double-blind placebo controlled fMRI study. Front Psychiatr 2010;1.

[22] Scantamburlo G, Hansenne M, Fuchs S, Pitchot W, Maréchal P, Pequeux C, et al. Plasma oxytocin levels and anxiety in patients with major depression. Psychoneuroendocrinology 2007;32:407-10.

[23] Scantamburlo G, Ansseau M, Geenen V, Legros JJ. Intranasal oxytocin as an adjunct to escitalopram in major depression. J Neuropsychiatry Clin Neurosci 2011;23:2.

[24] Spielberger CD, Gorsuch RL, Lushene R, Vagg PR, Jacobs GA. Manual for the state-trait anxiety inventory. Consulting psychologists' press. CA: Palo Alto; 1983.

[25] Turan T, Uysal C, Asdemir A, Kilic E. May oxytocin be a trait marker for bipolar disorder? Psychoneuroendocrinology 2013;38:2890-6.

[26] Uvnäs-Moberg K. Antistress pattern induced by oxytocin. News Physiol Sci 1998;13:22-5.

[27] van Ijzendoorn M, Bhandari R, van der Veen R, Grewen K, Bakermans-Kranen- burg MJ. Elevated salivary levels of oxytocin persist more than seven hours after intranasal administration. Front Neurosci 2012;6:174.

[28] Zink C, Meyer-Lindenberg A. Human neuroimaging of ocytocin and vasopressin in social cognition. Horm Behav 2012;61:400-9. 